\title{
Reduction of apoptosis by proanthocyanidin-induced autophagy in the human gastric cancer cell line MGC-803
}

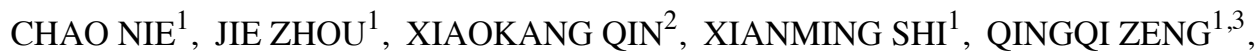 \\ JIA LIU $^{1}$, SHIHAI YAN ${ }^{3}$ and LEI ZHANG ${ }^{1}$ \\ ${ }^{1}$ Jiangsu Jiankang Vocational College; ${ }^{2}$ Nanjing KeyGEN Biotech Co., Ltd.; ${ }^{3}$ Affiliated Hospital of Nanjing \\ University of Chinese Medicine, Qinhuai, Nanjing, Jiangsu 210029, P.R. China
}

Received September 10, 2015; Accepted October 20, 2015

DOI: $10.3892 / o r .2015 .4419$

\begin{abstract}
Proanthocyanidins are flavonoids that are widely present in the skin and seeds of various plants, with the highest content in grape seeds. Many experiments have shown that proanthocyanidins have antitumor activity both in vivo and in vitro. Autophagy and apoptosis of tumor cells induced by drugs are two of the major causes of tumor cell death. However, reports on the effect of autophagy induced by drugs in tumor cells are not consistent and suggest that autophagy can have synergistic or antagonistic effects with apoptosis. This research was aimed at investigating whether proanthocyanidins induced autophagy and apoptosis in human gastric cancer cell line MGC-803 cells and to identify the mechanism of proanthocyanidins action to further determine the effect of proanthocyanidins-induced autophagy on apoptosis. MTT assay was used to examine the proanthocyanidin cytotoxicity against human gastric cancer cell line MGC-803. Transmission electron microscopy and monodansylcadaverine (MDC) staining were used to detect autophagy. Annexin V APC/7-AAD double staining and Hoechst 33342/propidium iodide (PI) double staining were used to explore apoptosis. Western blotting was used to determine expression of proteins related to autophagy and apoptosis. Real-time quantitative PCR technology was used to determine the mRNA level of Beclin1 and BCL-2. The results showed that proanthocyanidins exhibit a significant inhibitory effect on the human gastric cancer cell line MGC-803 proliferation in vitro and simultaneously activate autophagy and apoptosis to promote cell death. Furthermore, when proanthocyanidin-induced autophagy is inhibited, apoptosis increases significantly, proanthocyanidins can be used together with autophagy inhibitors to enhance cytotoxicity.
\end{abstract}

Correspondence to: Professor Lei Zhang, Jiangsu Jiankang Vocational College, 129 Hanzhong Road, Qinhuai, Nanjing, Jiangsu 210029, P.R. China

E-mail: jsjkzl@outlook.com

Key words: proanthocyanidins, gastric cancer, MGC-803, autophagy, apoptosis

\section{Introduction}

Gastric cancer is one of the most common malignant cancers. According to incomplete statistics, there are approximately one million new cases of gastric cancer diagnosed worldwide each year, and gastric cancer is the second leading cause of death, accounting for $\sim 8 \%$ of cancer-related deaths (1). Since the early symptoms of gastric cancer are not obvious, the cancer is typically in the middle and advanced stages by diagnosis, and its five-year survival rate is less than $20 \%$ (2). In 2006 alone, there were 950,000 new cases of gastric cancer, which accounted for $9 \%$ of new cancer cases, behind only lung, breast and colon cancer (3). Therefore, gastric cancer is a malignant tumor that seriously endangers human health. Approximately $70 \%$ of gastric cancers occur in developing countries, which have less medical resources than developed countries (4). More than $40 \%$ of patients with gastric cancer are Chinese (5). Although research is committed to the development of emerging fields such as nano-medicine (6-8) and stem cell technology (9-11), surgical resection, radiotherapy and chemotherapy are still the main treatment methods for malignant tumors at present. However, current chemotherapy drugs have many issues. Although these drugs are able to delay tumor growth and extend survival, they are highly controversial in tumor treatment since of the lack of an ideal therapeutic effect, the frequent occurrence of drug resistance, and strong toxic side-effects (12-14). Therefore, the extraction of highly effective, low-toxicity active ingredients from natural products to replace or combine with existing chemotherapy drugs has become a new research trend.

Proanthocyanidins are flavonoids that are widely present in the skin and seeds of various plants, with the highest content in grape seeds $(15,16)$. Their molecular formula is $\mathrm{C}_{30} \mathrm{H}_{26} \mathrm{O}_{12}$, and their molecular weight is $578.52 \mathrm{Da}$. Currently, proanthocyanidins can be extracted from many natural compounds and are also a major component of many Chinese medicines (17-19). Long-term studies have shown that proanthocyanidins possess anti-inflammatory properties, decrease blood pressure, and inhibit platelet aggregation, atherosclerosis and oxidation, among other functions (20-24). Since they are widely available and exhibit low toxicity and few side-effects, proanthocyanidins have received wide attention. In recent years, many experiments have shown that 
proanthocyanidins have antitumor activity both in vivo and in vitro, and proanthocyanidins were shown to inhibit or kill many types of tumors (25-29).

Autophagy and apoptosis of tumor cells induced by drugs are two of the major causes of tumor cell death. Autophagy is an important cellular metabolic process that is highly conserved throughout evolution and is widely present in eukaryotic cells. Autophagy is a programmed cell death that is different from apoptosis and is termed type II programmed cell death $(30,31)$. Autophagy is characterized by the presence of a large number of autophagosomes in the cytoplasm, and various digestive enzymes in the lysosome digest and degrade the contents in the vacuoles to convert them into substances required by the body for energy $(32,33)$. Several studies have confirmed that autophagy plays an important role in tumorigenesis and therapy (34-36). However, reports on the effect of autophagy induced by drugs in tumor cells are not consistent and suggest that autophagy can have synergistic or antagonistic effects with apoptosis $(37,38)$. In the present study, we treated the human gastric cancer cell line MGC-803 with proanthocyanidins to determine whether proanthocyanidins induced autophagy and apoptosis in these cells and to identify the mechanism of proanthocyanidins action to further determine the effect of proanthocyanidin-induced autophagy on apoptosis.

\section{Materials and methods}

Reagents. Proanthocyanidins were bought from Nanjing Zelang Medical Technology Co. Ltd. (Nanjing, China), with a purity of over $98 \%$ as testified by high-performance liquid chromatography. SDS-proanthocyanidins GE Gel Preparation kit glyceraldehyde-3-phosphate dehydrogenase (GAPDH) were purchased from Nanjing KeyGen Biology China (Nanjing, China), monodansylcadaverine (MDC) autophagy detection kit, Annexin V-APC/7-AAD apoptosis detection kit, First Strand cDNA Synthesis kit and Taq DNA polymerase were purchased from Sigma-Aldrich (St. Louis, MO, USA). Cytotoxicity assay kit, 3-methyladenine (3-MA), Hoechst 33342/propidium iodide (PI) double staining kit and MTT cell proliferation were purchased from Thermo Fisher Scientific (Shanghai, China).

Cell culture. The human gastric cancer cell line MGC-803 was purchased from Nanjing KeyGen Biology China. The cells were cultured in RPMI-1640 complete medium containing $10 \%$ calf serum $(\mathrm{CS})$ at $37^{\circ} \mathrm{C}$ in a $5 \% \mathrm{CO}_{2}$ incubator. Cells in the logarithmic growth phase were used for the experiments.

MTT assay for cell proliferation $\left(I C_{50}\right)$. A cell suspension with a concentration of $5 \times 10^{4}$ cells $/ \mathrm{ml}$ was prepared, and $100 \mu \mathrm{l}$ of the cell suspension was added to each well of a 96-well culture plate, incubated at $37^{\circ} \mathrm{C}$ in a $5 \% \mathrm{CO}_{2}$ incubator (Sanyo XD-101; Sanyo, Osaka, Japan) for $24 \mathrm{~h}$. Complete medium was used to dilute the drug to the desired concentrations (400, 200, 100, $50,25,12.5,6.25,3.125,1.5625$ and $0.78125 \mu \mathrm{g} / \mathrm{ml})$, and $100 \mu \mathrm{l}$ of the corresponding drug-containing medium was added to each well. A negative control and a positive control group were also included. The 96 -well plate was incubated at $37^{\circ} \mathrm{C}$ in a $5 \%$ $\mathrm{CO}_{2}$ incubator (Sanyo XD-101) for $48 \mathrm{~h}$. The plate was then subjected to MTT staining, and the OD value was measured at $\lambda=490 \mathrm{~nm}$. The inhibition rate and drug $\mathrm{IC}_{50}$ value of each group were calculated.

Annexin V APC/7-AAD double staining to detect apoptosis. Cells growing in the logarithmic phase were trypsinized and seeded into a 6 -well plate. The corresponding drugcontaining medium was added $(100,20$ or $4 \mu \mathrm{g} / \mathrm{ml})$ after the cells were attached to the plate and negative control group was included at the same time. After treatment with the drug for $48 \mathrm{~h}, 0.25 \%$ trypsin (without EDTA) was used to trypsinize and gather the cells. The cells were washed twice with phosphate-buffered saline (PBS) (centrifugation at $2,000 \mathrm{rpm}, 5 \mathrm{~min}$ ), and $5 \times 10^{5}$ cells were collected. The cells were then resuspended in $500 \mu \mathrm{l}$ of binding buffer. After $5 \mu \mathrm{l}$ of Annexin V-APC was added and mixed well, $5 \mu \mathrm{l}$ of 7-AAD was added and mixed well. The reaction was performed at room temperature for 5-15 min in the dark, and a flow cytometer (FACSCalibur; Becton-Dickinson, USA) was used to detect apoptosis.

Transmission electron microscopy. MGC-803 cells in the logarithmic growth phase were incubated in drug-containing medium $(100,20$ and $4 \mu \mathrm{g} / \mathrm{ml})$. A negative control group was included at the same time. All the cells were harvested $24 \mathrm{~h}$ later. Trypsin $(0.25 \%)$ was used to remove the cells from the plate. The cells were then centrifuged at 1,000 rpm for $10 \mathrm{~min}$. After the supernatant was discarded, the cells were washed twice with PBS, and $2.5 \%$ glutaric acid was added. The cells were fixed for $90 \mathrm{~min}$ at $4^{\circ} \mathrm{C}$. After the cells were embedded, sectioned and stained with uranyl acetate and lead citrate, the autophagosomes were observed under a transmission electronic microscope (JEM-1011, Japan).

MDC staining to detect autophagy. Cells in the logarithmic growth phase were trypsinized and seeded into a 6-well plate. The next day, after the cells attached to the walls, drug-containing medium was added $(100,20$ and $4 \mu \mathrm{g} / \mathrm{ml})$. A negative control group was included at the same time. After treatment with the drug for $48 \mathrm{~h}, 0.25 \%$ trypsin (without EDTA) was used to gather the cells. Wash buffer $(1 \mathrm{X} ; 300 \mu \mathrm{l})$ was used to wash the cells once, and an appropriate amount of $1 \mathrm{X}$ wash buffer was added to resuspend the cells, with the cell concentration adjusted to $10^{6}$ cells $/ \mathrm{ml}$. A total of $90 \mu \mathrm{l}$ of cell suspension was transferred to a new microfuge tube and $10 \mu \mathrm{l}$ of MDC staining solution was added and gently mixed. After staining at room temperature for 15-45 min in the dark, the cells were gathered by centrifugation at $800 \mathrm{x}$ g for $5 \mathrm{~min}$. Wash buffer was used to wash the cells three times, and the cells were resuspended in $100 \mu \mathrm{l}$ of gatherion buffer. The cell suspension was dropped onto a slide and covered with a coverslip. The slide was then observed under a fluorescence microscope (Olympus IX51; Olympus, Japan).

Western blotting to determine protein expression. Cells in the logarithmic growth phase were trypsinized and seeded onto a 6-well plate. The next day, after the cells attached, drug-containing medium was added (100, 20 and $4 \mu \mathrm{g} / \mathrm{ml})$. A negative control group was included at the same time. Pre-chilled lysis buffer $(200 \mu \mathrm{l})$ was added to each group. 
After mixing, the lysate was incubated on ice for $30 \mathrm{~min}$. After vortexing, the lysate was centrifuged at $13,000 \mathrm{x} \mathrm{g}$ for $10 \mathrm{~min}$ at $4^{\circ} \mathrm{C}$. The supernatant was saved, and the BCA method was used to measure the protein concentration of the samples. The proteins were resolved on a $10 \%$ SDS-PAGE gel and transferred to a PVDF membrane. After the membrane was blocked overnight with $5 \%$ non-fat milk, the primary antibody (1:200) was added and incubated overnight at $4^{\circ} \mathrm{C}$ in a sealed bag. TBST was used to wash the membrane three times for $10 \mathrm{~min}$, and the membrane was then incubated with the secondary antibody $(1: 4,000)$ for $1 \mathrm{~h}$. Finally, the membrane was incubated with chemiluminescence solution and exposed to film.

Hoechst 33342/PI double staining to detect apoptosis. Cells in the logarithmic growth phase were trypsinized and seeded into a 6-well plate. The next day, after the cells attached, drug-containing medium was added. A negative control group was included at the same time. After treatment with the drug for $48 \mathrm{~h}, 0.25 \%$ trypsin (without EDTA) was used to gather the cells. A total of $10^{5}-10^{6}$ cells was resuspended in $1 \mathrm{ml}$ of medium, $10 \mu \mathrm{l}$ of Hoechst 33342 staining solution was added to the cells and mixed well, and the suspension was incubated at $37^{\circ} \mathrm{C}$ for $5-15 \mathrm{~min}$. The cells were centrifuged at $500-1,000 \mathrm{rpm}$ for $5 \mathrm{~min}$ at $4^{\circ} \mathrm{C}$, and the supernatant was discarded. Buffer A (1 ml) was used to resuspend the cells, and $5 \mu \mathrm{l}$ of PI staining solution was added, and incubated at room temperature for 5-15 $\mathrm{min}$ in the dark. The suspension was mixed well and observed under a fluorescence microscope Olympus IX51.

Fluorescence quantitative PCR to detect gene expression. Total RNA was isolated from logarithmically growing MGC-803 cells, and the purity of the RNA was determined. The isolated RNA was reverse transcribed into cDNA using a kit from Thermo Fisher. Fluorescent staining and a quantitative PCR were used to perform real-time quantitative PCR (ABI StepOne Plus, USA). The primers were synthesized by Nanjing GenScript Technology Co., Ltd. with the sequences: GAPDH (101-bp product) [5'-ACAACTTTGGTATCGTGG AAGG-3' (sense), and 5'-GCCATCACGCCACAGTTTC-3' (antisense)]; Beclin1 [(140-bp product) (5'-ATGTCCACAGA AAGTGCCAA-3' (sense), and 5'-GGGTGATCCACATCTGT CTG-3' (antisense)]; and BCL-2 (114-bp product) [(5'-AAATC CGACCACTAATTGCC-3' (sense), and 5'-TGCTCTTCAGAT GGTGATCC-3' (antisense)]. The amplification conditions were $95^{\circ} \mathrm{C}$ pre-denaturation for $5 \mathrm{~min}$ followed by $95^{\circ} \mathrm{C}$ denaturation for $15 \mathrm{sec}, 60^{\circ} \mathrm{C}$ annealing for $20 \mathrm{sec}$, and $72^{\circ} \mathrm{C}$ extension for $40 \mathrm{sec}$ for a total of 40 cycles. The specificity of the amplified products was monitored by melting curves. Software was used to calculate the relative expression of the target genes in each group, and GAPDH was used as an internal reference to assess the expression of target genes.

Statistical methods. The data are presented as the mean \pm standard deviation. The SPSS 16.0 statistical software was used for data analysis. Analysis of variance (ANOVA) was used to compare the difference between groups under different conditions, and $\mathrm{p}<0.05$ was considered to indicate a statistically significant result.

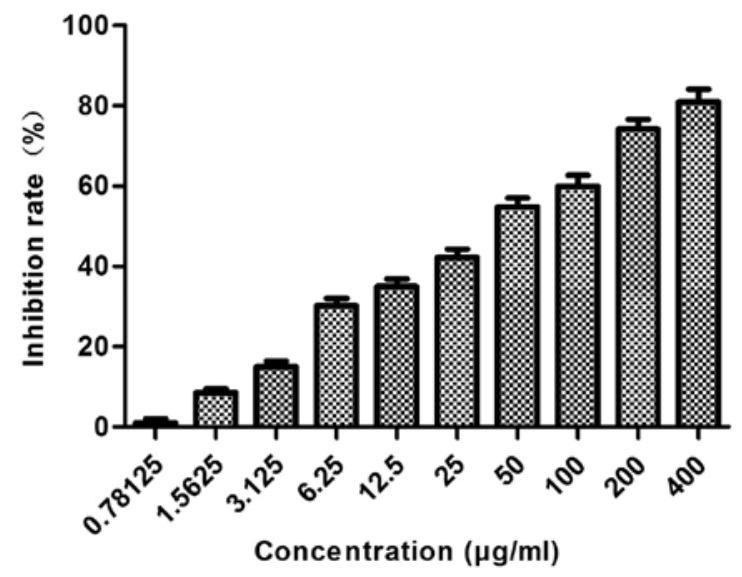

Figure 1. Concentration curve of the inhibition of MGC-803 cell proliferation by proanthocyanidins. A range of concentrations of proanthocyanidins was used to treat MGC-803 cells for $48 \mathrm{~h}$, and a dose-dependent inhibition of cell proliferation was observed. The data are presented as the mean \pm standard deviation $(n=6)$.

\section{Results}

The inhibitory effect of proanthocyanidins on the proliferation of MGC-803 cells. As shown in Fig. 1, a range of concentrations of proanthocyanidins $(400,200,10,50,25,12.5,6.25$, $3.125,1.5625$ and $0.78125 \mu \mathrm{g} / \mathrm{ml}$ ) was used to treat MGC-803 cells for $48 \mathrm{~h}$, and the results showed that proanthocyanidins inhibited MGC-803 cell proliferation in a dose-dependent manner. At $48 \mathrm{~h}$, the $\mathrm{IC}_{50}$ value was $40.654 \mu \mathrm{g} / \mathrm{ml}$.

The effect of proanthocyanidins on the apoptosis of MGC-803 cells. To test whether the inhibition of cell proliferation by proanthocyanidins was associated with apoptosis, MGC-803 cells were treated with proanthocyanidins for $48 \mathrm{~h}$, and then, flow cytometry was used to analyze the percentage of apoptotic cells. The results in Fig. 2 show that proanthocyanidins induced apoptosis in MGC-803 cells in a dose-dependent manner.

The effect of proanthocyanidins on the microstructural morphology of MGC-803 cells. To verify whether the cytoplasmic vacuoles observed by inverted microscopy were related to autophagy, transmission electron microscopy was used to observe autophagosomes in MGC-803 cells treated with proanthocyanidins. As shown in Fig. 3, untreated cells had normal nuclei, cytoplasm and organelles, whereas proanthocyanidin-treated cells showed a high number of autophagosomes of various sizes, and autophagosomes containing mitochondria were also observed by electron microscopy. This suggests that autophagy occurred in the cells after proanthocyanidin treatment.

MDC staining for autophagosome labeling. An inverted fluorescence microscope was used to observe MDC-labeled autophagic vacuoles, clear punctate structures were observed in the cytoplasm and perinuclear region, and the changes of the particles inside the cell were used to determine the level of autophagy. As shown in Fig. 4, compared with the control group, proanthocyanidins-treated cells showed stronger 

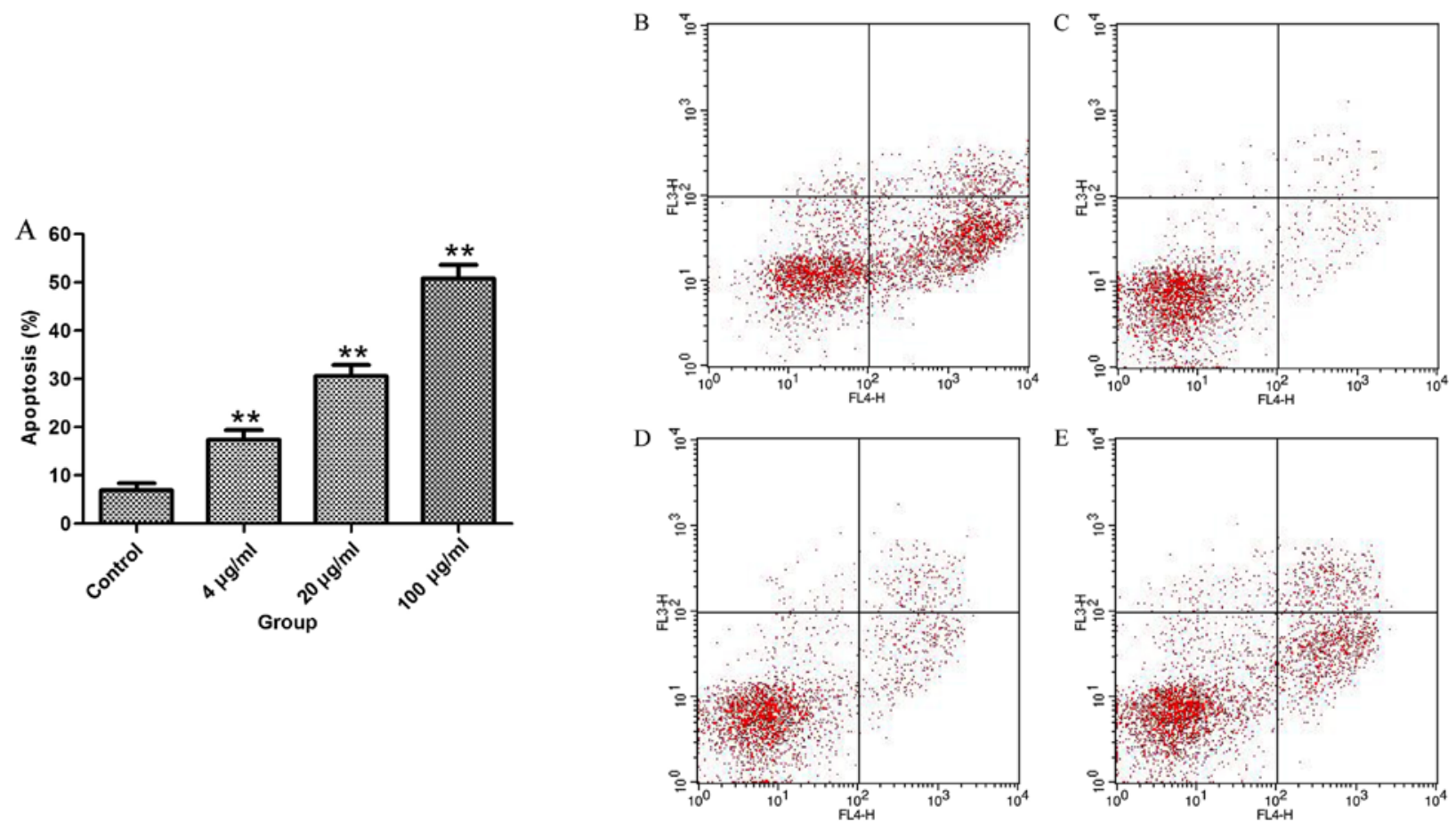

Figure 2. Curve of the effect of different concentrations of proanthocyanidins on the apoptosis of MGC-803 cells. (A) Compared with the control group, the percentage of apoptotic cells in the proanthocyanidins group significantly increased in a dose-dependent manner. The data are presented by the mean \pm standard deviation (n=3). ${ }^{* *} \mathrm{p}<0.01$. (B-E) Apoptotic profile of cells treated with different concentrations of proanthocyanidins $(0,4,20$ and $100 \mu \mathrm{g} / \mathrm{ml})$.

A

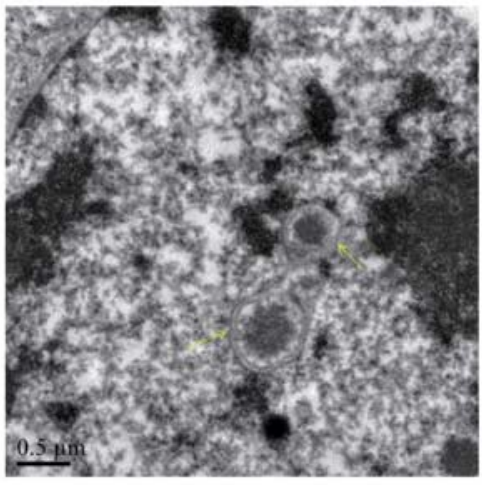

$\mathrm{C}$

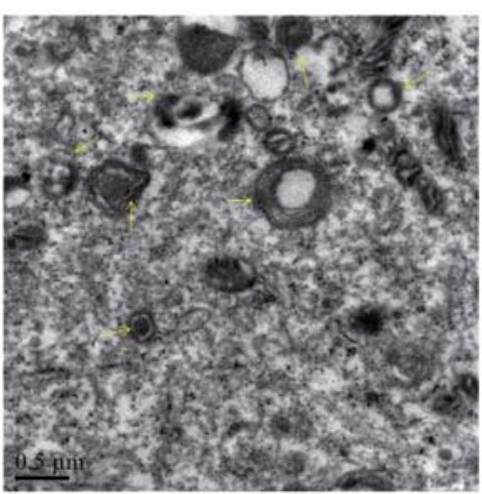

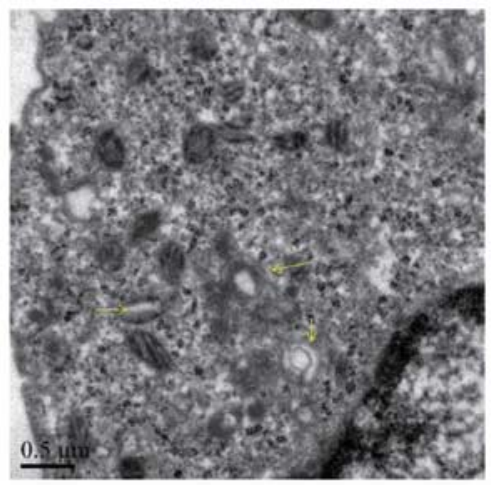

D

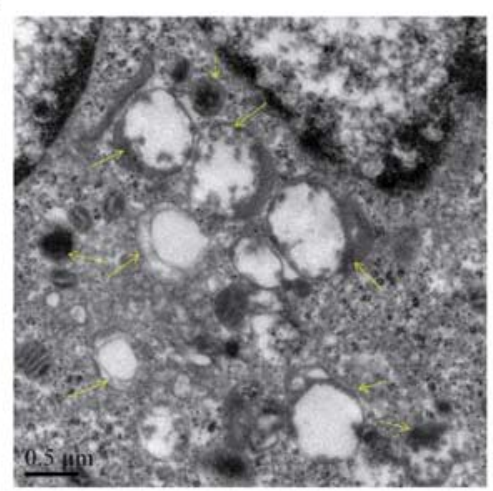

Figure 3. (A) Observation of the microstructure of MGC-803 cells treated with proanthocyanidins. Untreated MGC-803 cells had a normal nucleus, cytoplasm and organelle morphology. (B-D) Cells treated with different concentrations of proanthocyanidins $(4,20$ and $100 \mu \mathrm{g} / \mathrm{ml}) \mathrm{showed}$ a high number of various sizes of autophagic vacuoles, and some autophagosomes contained mitochondria and cytoplasm. (A-D, magnification, x20,000). The arrows indicate autophagic vacuoles and autophagosomes.

fluorescence intensity and more autophagic vacuoles labeled with MDC, and the number and staining intensity of the vacuoles increased with increasing proanthocyanidins dose. This suggests that proanthocyanidins induced autophagy. 
A

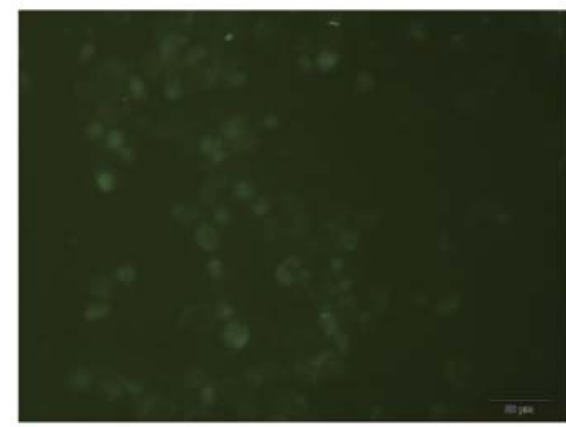

$\mathrm{C}$

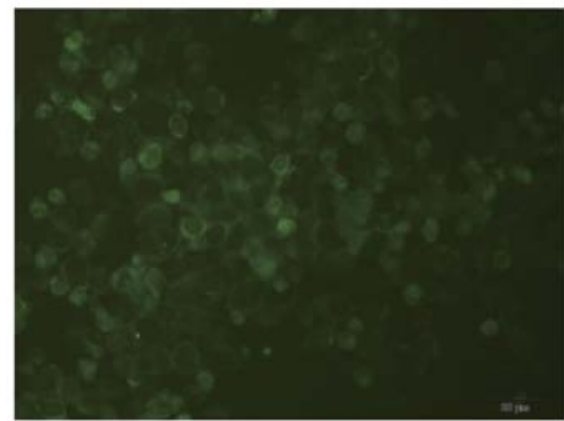

B

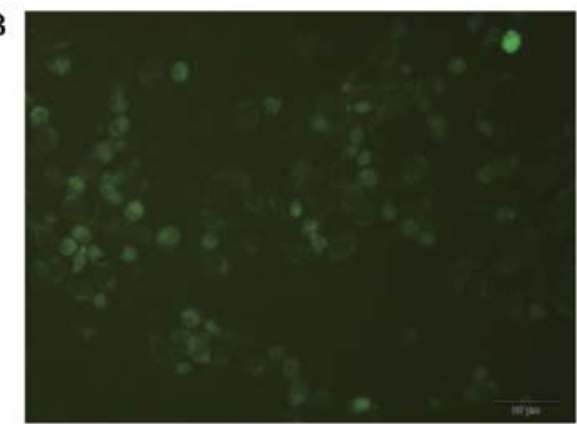

$\mathrm{D}$

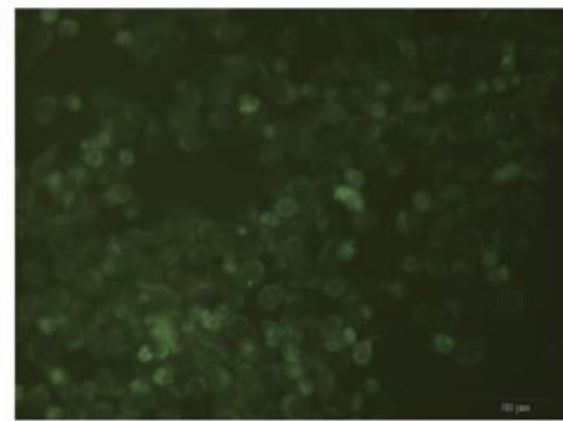

Figure 4. MGC-803 autophagic vacuoles labeled with MDC. Compared with the control group (B-D; magnification, x100), cells treated with different concentrations of proanthocyanidins (4,20 and $100 \mu \mathrm{g} / \mathrm{ml}$ ) exhibited increased number and fluorescence intensity of autophagic vacuoles labeled by MDC. (A) $0 \mu \mathrm{g} / \mathrm{ml}$, magnification, $\mathrm{x} 100$.

$\mathbf{A}$

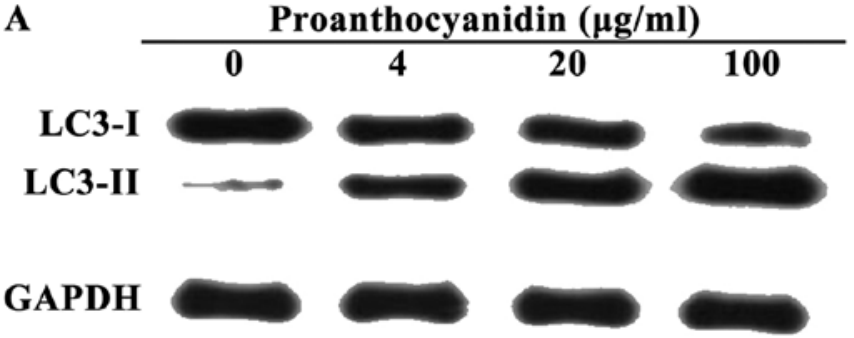

Proanthocyanidin $(\mu \mathrm{g} / \mathrm{ml})$
B Proanthocyanidin $(\mu \mathrm{g} / \mathrm{ml})$

Caspase 9

$\begin{array}{llll}0 & 4 & 20 & 100\end{array}$

GAPDH

Figure 5. Proanthocyanidins induce the expression of LC3 and caspase 9 in MGC-803 cells. (A) Western blotting of the LC3 protein level in MGC-803 cells treated with different concentrations of proanthocyanidins. (B) Western blotting of the caspase 9 protein level in MGC-803 cells treated with different concentrations of proanthocyanidins.

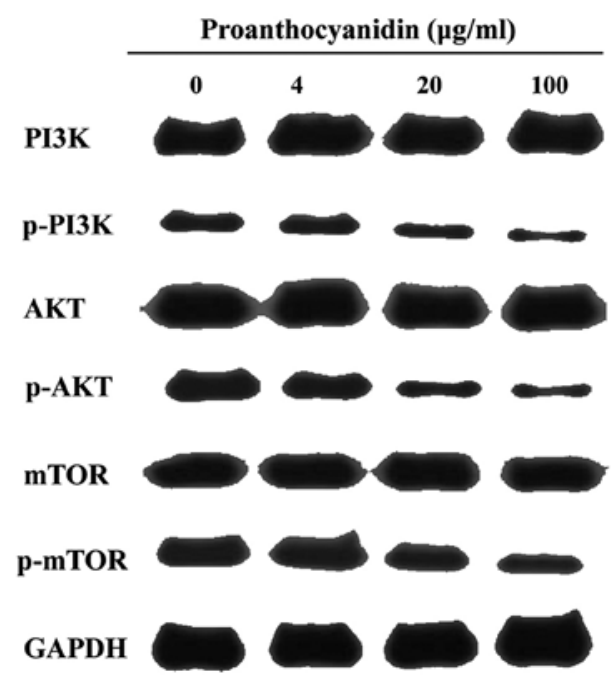

Figure 6. The effect of proanthocyanidins on the PI3K/AKT/mTOR signaling pathway. After proanthocyanidins treatment, the expression of PI3K, AKT, and mTOR did not significantly change, but the phosphorylation of PI3K, AKT and mTOR decreased in a dose-dependent manner. ${ }^{* *} \mathrm{p}<0.01$.
Proanthocyanidins induces LC 3 and caspase 9 expression in $M G C-803$ cells. Western blot analyses were used to detect changes in the levels of LC3 and caspase 9 after MGC-803 cells were treated with proanthocyanidins. On SDS-PAGE gels, LC3-II ran faster than LC3-I, producing two bands by western blotting. Fig. 5A shows that the untreated cells exhibited only a faint LC3-I band, whereas the LC3-II band was not detected. In contrast, after treatment with proanthocyanidins, the level of LC3-II increased significantly in a dose-dependent manner. Fig. 5B shows that treatment with proanthocyanidins significantly increased the expression of caspase 9 compared with the control group in a dose-dependent manner.

Effect of proanthocyanidins on the phosphatidylinositol 3 kinase $(P I 3 K) /$ protein kinase B $(P K B / A K T) /$ mammalian target of rapamycin (mTOR) signaling pathway. The PI3K/AKT/mTOR signaling pathway is the canonical pathway that negatively regulates the initiation of autophagy. It has been reported that inhibition of this pathway induces cell autophagy. As shown in Fig. 6, western blot analyses showed 


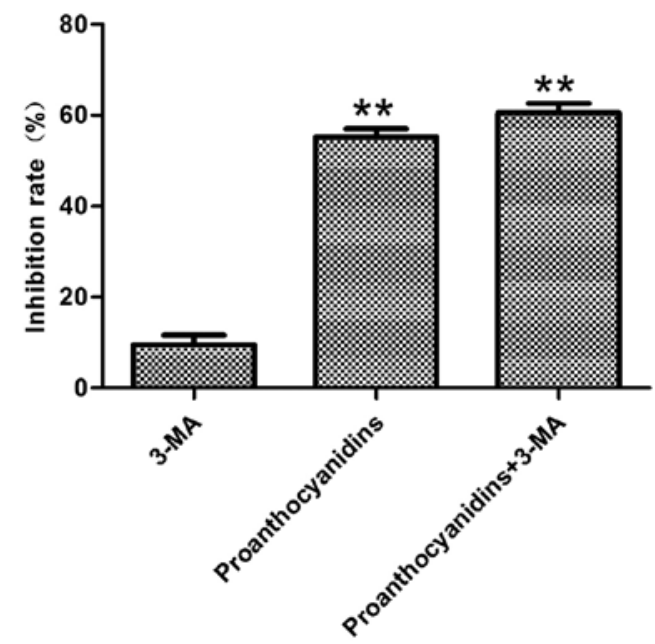

Figure 7. Proanthocyanidin cytotoxicity following inhibition of autophagy. MGC-803 cells were pretreated with 3-MA $(5 \mathrm{mM})$ for $48 \mathrm{~h}$, and the cells were then treated with proanthocyanidins $(40.7 \mu \mathrm{g} / \mathrm{ml})$ for $48 \mathrm{~h}$. MTT assays were used to detect cell proliferation. The inhibition of cell proliferation by proanthocyanidins after the addition of 3-MA was significantly increased when compared with cells treated with proanthocyanidins alone. Data are expressed as the mean \pm standard deviation $(\mathrm{n}=6)^{* *} \mathrm{p}<0.01$.

that proanthocyanidins inhibited the phosphorylation of PI3K, AKT and mTOR in the PI3K/AKT/mTOR signaling pathway.

Inhibition of autophagy increased the cytotoxicity of proanthocyanidins. Preliminary experiments determined that proanthocyanidin treatment of MGC- 803 cells activated both autophagy and apoptosis and that the inhibition of MGC-803 cell proliferation by proanthocyanidins occurred in a dosedependent manner. To understand whether the cytotoxicity exhibited by proanthocyanidins was mediated by autophagy, the autophagy inhibitor 3-MA was added, and MTT assays were used to examine its cytotoxicity. The results showed that compared with cells treated with only proanthocyanidins, the addition of 3-MA significantly increased the percentage of apoptotic MGC-803 cells (Fig. 7).

Hochest 33342 and PI double fluorescence staining of live cells. Hochest 33342 and PI double staining is able to distinguish live and dead cells. When cells are in the late apoptotic stage or in the early necrotic stage, the nuclei are red in color, whereas the nuclei of live cells are blue. As shown in Fig. 8, MGC-803 cells treated with proanthocyanidins for $48 \mathrm{~h}$ exhibited nuclei with a bead-like shape, forming apoptotic bodies. There was no significant difference between treatment with 3-MA alone (Fig. 8B) and control cells (Fig. 8A). However, cells treated with proanthocyanidins (Fig. 8C) exhibited an increased percentage of apoptotic cells compared with the control cells (Fig. 8A) (p<0.001). Cells treated with proanthocyanidins + 3-MA (Fig. 8D) showed an increased percentage of apoptotic cells compared with cells treated with proanthocyanidins alone (Fig. 8C) $(\mathrm{p}<0.01)$. These results showed that apoptosis increased significantly in response to the inhibition of autophagy induced by proanthocyanidins.

The effect of proanthocyanidins on apoptosis following inhibition of autophagy. Since we found that the cytotoxicity
A

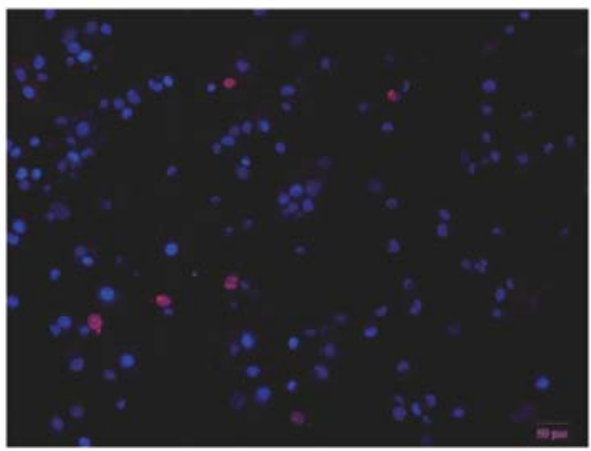

$\mathrm{C}$

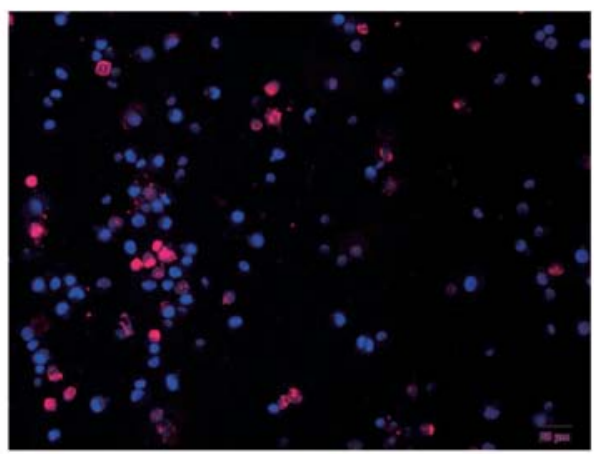

B
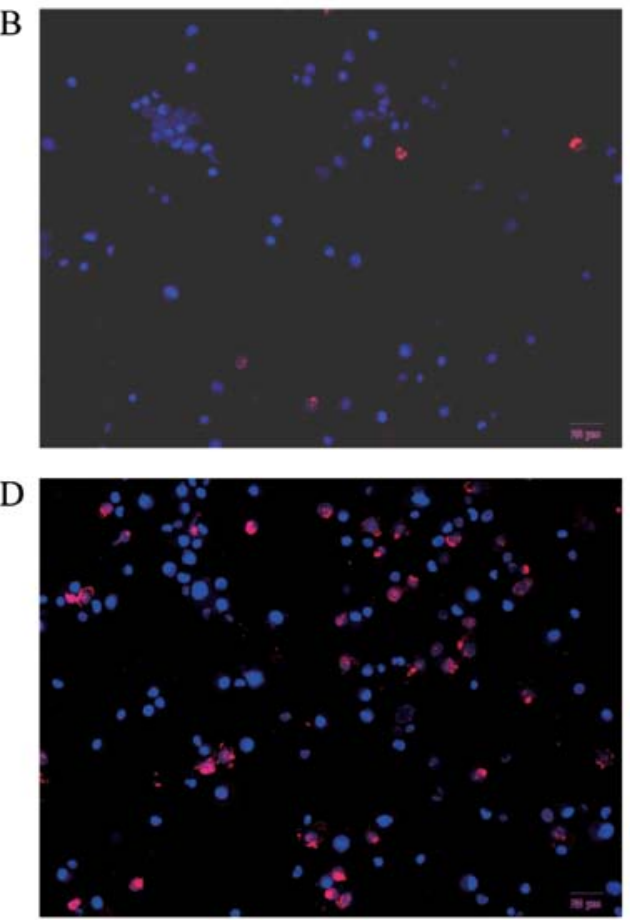

Figure 8 . Hoechst and PI double fluorescence staining of live cells. After MGC-803 cells were treated with proanthocyanidins for 48 h, the nuclei showed a bead-like shape, forming apoptotic bodies. The percentage of apoptotic cells was $5.76 \pm 0.01 \%$ for the controls (A) and $34.42 \pm 0.02 \%$ for cells treated with proanthocyanidins (C). Compared with the controls, the percentage of apoptotic proanthocyanidin-treated cells increased significantly (p<0.01). The percentage of apoptotic 3-MA-treated cells (B) was $6.78 \pm 0.02 \%$; compared with the control cells, there was no significant difference. The percentage of cells treated with proanthocyanidins + 3-MA (D) was $48.57 \pm 0.04 \%$, which was significantly increased compared with cells treated with proanthocyanidins alone (p<0.01). Data are expressed as the mean \pm standard deviation $(\mathrm{n}=3)$. 

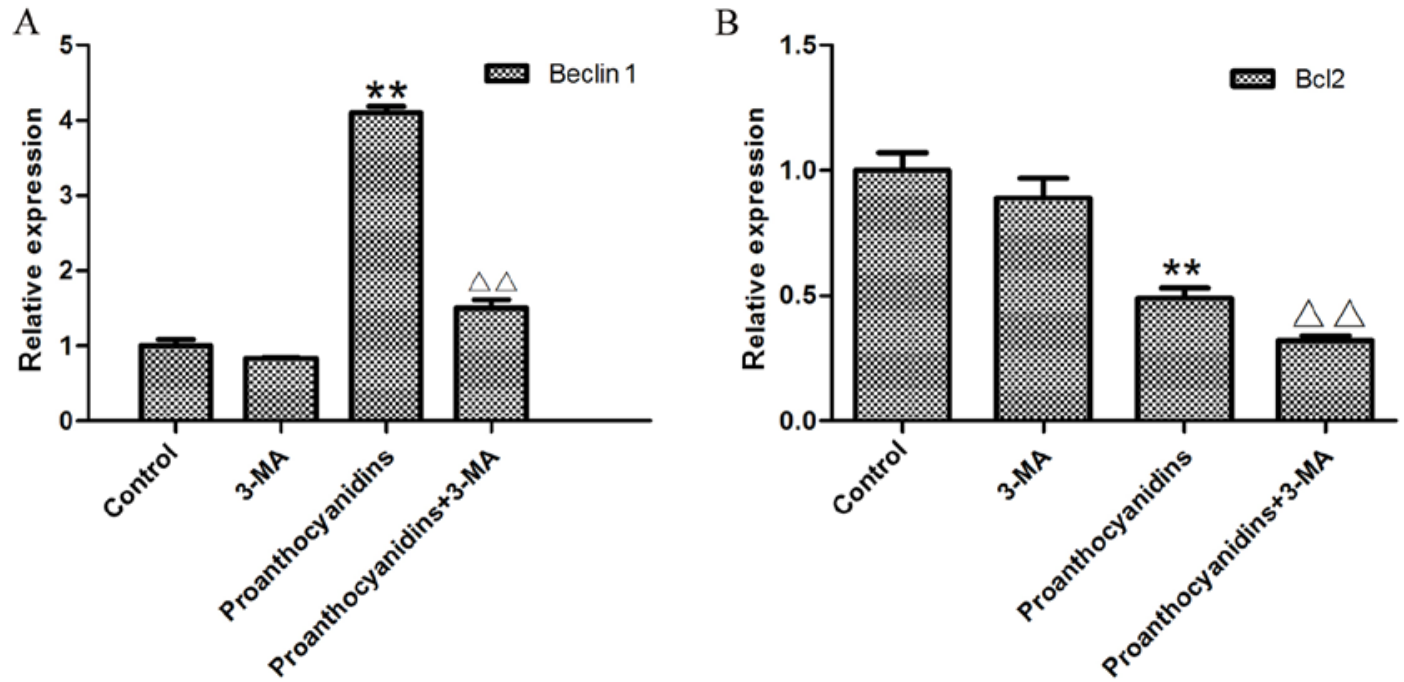

Figure 9. The effect of proanthocyanidins on autophagy and apoptosis when autophagy was inhibited. After treatment with 5 mM 3-MA, the expression of Beclin1 was decreased compared with that in control cells. After treatment with $40.7 \mu \mathrm{g} / \mathrm{ml}$ proanthocyanidins, the expression of Beclin1 increased significantly compared with that in control cells, ${ }^{* *} \mathrm{p}<0.01$. However, when the proanthocyanidins were added after treatment with 3 -MA, Beclin1 expression was significantly reduced compared with that in cells treated with proanthocyanidins alone, ${ }^{\Delta} \mathrm{p}<0.01$. (A) Data are expressed as the mean \pm standard deviation $(\mathrm{n}=3)$. After treatment with proanthocyanidins, the level of $\mathrm{Bcl} 2$ decreased significantly compared with that in control cells, ${ }^{* *} \mathrm{p}<0.01$, whereas simultaneous addition of 3-MA significantly decreased Bcl2 expression compared with that in cells treated with proanthocyanidins alone, ${ }^{\Delta \Delta} \mathrm{p}<0.01$. (B) Data are expressed as the mean \pm standard deviation $(\mathrm{n}=3)$.

of proanthocyanidins was increased after the inhibition of autophagy, we speculated whether autophagy inhibited the effect of proanthocyanidins on apoptosis. Real-time quantitative PCR was used to investigate MGC-803 cells treated with proanthocyanidins. Using Beclin1 and BCL-2 as indicators, we investigated the effect of proanthocyanidins on autophagy and apoptosis when autophagy was inhibited. As shown in Fig. 9, after the addition of $5 \mathrm{mM} 3-\mathrm{MA}$, the expression of Beclin1 decreased compared with that in control cells. After treatment with $40.7 \mu \mathrm{g} / \mathrm{ml}$ proanthocyanidins, the expression of Beclin1 increased significantly compared with that in control cells. However, when the proanthocyanidins were added after treatment with 3-MA, Beclin1 expression was significantly decreased compared with the level in cells treated with proanthocyanidins alone. After treatment with proanthocyanidins, BCL-2 decreased significantly compared with expression in the control cells, whereas the addition of 3-MA significantly decreased BCL-2 expression compared with that in cells treated with proanthocyanidins alone. Thus, when autophagy was inhibited, the apoptotic effect of proanthocyanidins was increased.

\section{Discussion}

In the present study, MTT assays were used to determine the effect of proanthocyanidins on the human gastric cancer MGC-803 cells and to calculate the $\mathrm{IC}_{50}$. The results showed that proanthocyanidins significantly inhibit MGC-803 cells in a dose-dependent manner.

To test whether the inhibition of MGC- 803 cells by proanthocyanidins is related to the induction of autophagy, we used MDC staining to label autophagic vacuoles and transmission electronic microscopy to observe autophagosomes, which are published methods to confirm the presence of autophagy (39). MDC staining showed that proanthocyanidin-treated cells exhibited increased autophagic vacuoles, and transmission electronic microscopy confirmed the existence of autophagosomes in the cells. Therefore, from the morphology, we can preliminarily confirm that proanthocyanidins induce autophagy in MGC-803 cells. Microtubule-associated protein 1 light chain 3 (LC3) plays a key role in autophagy in mammalian cells. LC 3 consists of soluble LC3-I and lipidated LC3-II. Under various stresses, such as hypoxia and drug treatment, the cells initiate autophagy, and LC3-I undergoes a ubiquitination-like modification and processing to form LC3-II. Therefore, the level of LC3-II is positively correlated with the number of autophagic vacuoles. When autophagy occurs inside the cells, LC3-II increases significantly, and the detection of the change in LC3-II levels can accurately determine the amount of autophagy (40-43). When western blot analyses were used to determine the levels of the autophagy marker LC3, LC3-I levels decreased, whereas LC3-II increased in proanthocyanidin-treated MGC-803 cells, and both of these effects were dose-dependent. This confirmed that treatment with proanthocyanidin-induced autophagy in MGC-803 cells.

We next used flow cytometry to observe apoptosis of MGC-803 cells after treatment with proanthocyanidins. The proanthocyanidins significantly induced apoptosis in MGC-803 cells. According to published studies, proanthocyanidins induce apoptosis in multiple tumor cell types, and the above result is consistent with these reports $(44,45)$. Apoptosis is initiated by $\mathrm{p} 53$, which activates relevant proteins to form a channel on the mitochondria to allow cytochrome $c$ in the mitochondria to be released into the cytoplasm, which eventually activates caspase family proteases (46). Caspase family proteases are a type of aspartate-specific cysteine-containing proteases that are important for apoptosis. Caspases are 
divided into apoptosis initiation factors and apoptosis effectors according to their function (47). Caspase 9 is an important initiation factor and can be activated by other proteins or by itself to activate a series of downstream effectors. Eventually, the cells undergo biochemical and morphological changes that lead to apoptosis (48). Our experiments have shown that proanthocyanidins activate caspase 9 and induce apoptosis in MGC-803 cells.

Molecular signaling pathways are closely involved in autophagy and apoptosis. Phosphatidylinositol 3 kinase (PI3K)/ protein kinase $\mathrm{B}(\mathrm{PKB} / \mathrm{AKT}) /$ mammalian target of rapamycin (mTOR) is one of the currently most-studied pathways. This pathway is well accepted as being associated with cell autophagy and apoptosis. PI3K phosphorylates phosphatidylinositol $(4,5)$ bisphosphate [PtdIns $(4,5) \mathrm{P} 2]$ in the cytoplasmic membrane to generate phosphatidylinositol (3-5) triphosphate [PtdIns $(3,4,5)$ P3], which recruits AKT to the inner side of the cytoplasmic membrane. AKT is then phosphorylated and activated by another protein kinase, 3-phosphoinositide-dependent protein kinase 1 (PDK1). Activated AKT further activates mTOR by inhibiting the tuberous sclerosis complex ( $\mathrm{TSC} 1 / 2)$, which is an inhibitor of mTOR. The inhibition of TSC1/2 activity by phosphorylated AKT leads to the activation of mTOR. mTOR is a serine/threonine kinase that inhibits autophagy when activated (49). Similarly, apoptosis is also affected by the PI3K/AKT pathway. Activated AKT binds to Ser184 of the BCL-2 family member BAX. After phosphorylation, BAX inactivates mitochondrial cytochrome $c$, blocking the activation of caspases and inhibiting apoptosis (50). Whereas, activated AKT also phosphorylates Ser196 of caspase 9, inactivating it and thus directly reducing apoptosis (51). Multiple studies have shown that autophagy and apoptosis induced by a variety of drugs are all associated with the PI3K/AKT/ mTOR pathway (52-55). Western blot analyses showed that although proanthocyanidins did not affect the total amount of PI3K, AKT, and mTOR, these compounds did significantly reduce the amount of $\mathrm{p}-\mathrm{PI} 3 \mathrm{~K}, \mathrm{p}-\mathrm{AKT}$ and $\mathrm{p}$-mTOR; in other words, proanthocyanidins reduced the activation of the PI3K/ AKT/mTOR pathway. This may be one of the reasons that proanthocyanidins induce autophagy and apoptosis and is also consistent with other reports (56).

The results above showed that both autophagy and apoptosis are activated when proanthocyanidins induce cell death in the human gastric cancer MGC-803 cells and that the activation mechanism is associated with the inhibition of the $\mathrm{PI} 3 \mathrm{~K} / \mathrm{AKT} / \mathrm{mTOR}$ pathway.

To further explore the relationship between proanthocyanidin-induced autophagy and apoptosis, we used the classical autophagy inhibitor 3-methyladenine (3-MA) to determine the effect of autophagy on apoptosis. MTT assays found that after the addition of 3-MA, the effect of proanthocyanidins increased. When Hoechst 33342/PI double fluorescence staining was used to observe apoptosis after the inhibition of autophagy, apoptosis increased significantly. These results suggest that the inhibition of autophagy may increase apoptosis. To verify this hypothesis, real-time quantitative PCR technology was used to determine the mRNA level of Beclin1 and BCL-2. Beclin1 plays a key role in autophagy of mammalian cells. Beclin1 is also the mammalian homologue of the yeast protein ATG6/Vps30 and is located on human chromosome 17q21 (57). Beclin1 promotes the formation of autophagosomes by forming a complex with class III PI3K (58). The expression of Beclin1 is positively correlated with autophagy in multiple malignant tumor cell types (59-61). BCL-2 is a key regulator of the known apoptosis proteins and is negatively correlated with apoptosis induced by various signals $(62,63)$. When BCL-2 increases, the BCL-2/BAX heterodimer interferes with the release of cytochrome $c$, thereby blocking the activation of the upstream caspase protease and in turn inhibiting apoptosis (64-66). The inhibition of autophagy by 3-MA not only decreased Beclin1 mRNA but also increased BCL-2 mRNA, which further confirmed that apoptosis increases significantly when autophagy induced by proanthocyanidins is blocked.

In summary, proanthocyanidins exhibit a significant inhibitory effect on human gastric cancer cell (MGC-803) proliferation in vitro and simultaneously activate autophagy and apoptosis to promote cell death. The mechanism is associated with interference of the PI3K/AKT pathway by proanthocyanidins and a change in the amount of the downstream autophagy proteins LC3 and Beclin1, as well as in the apoptosis proteins BCL-2 and caspase 9. Furthermore, when proanthocyanidin-induced autophagy is inhibited, apoptosis increases significantly and tumor cells undergo cell death. Therefore, as an active ingredient of natural products with low toxicity, proanthocyanidins can be used together with autophagy inhibitors to enhance cytotoxicity.

\section{Acknowledgements}

The present study was supported by the State Administration of Traditional Chinese Medicine of Jiangsu Province (grant no. LZ13240).

\section{References}

1. Ren JS, Kamangar F, Qiao YL, Taylor PR, Liang H, Dawsey SM, Liu B, Fan JH and Abnet CC: Serum pepsinogens and risk of gastric and oesophageal cancers in the General Population Nutrition Intervention Trial cohort. Gut 58: 636-642, 2009.

2. No authors listed: Survival of Cancer Patients in Europe: The EUROCARE-2 study. IARC Sci Publ 151: 1-572, 1999.

3. Crew KD and Neugut AI: Epidemiology of gastric cancer. World J Gastroenterol 12: 354-362, 2006.

4. Jemal A, Bray F, Center MM, Ferlay J, Ward E and Forman D: Global cancer statistics. CA Cancer J Clin 61: 69-90, 2011.

5. Zhang J, Li Y, Chen X, Liu T, Chen Y, He W, Zhang Q and Liu S: Autophagy is involved in anticancer effects of matrine on SGC-7901 human gastric cancer cells. Oncol Rep 26: 115-124, 2011.

6. Kawasaki ES and Player A: Nanotechnology, nanomedicine, and the development of new, effective therapies for cancer. Nanomedicine 1: 101-109, 2005.

7. Valentini F, Carbone M and Palleschi G: Carbon nanostructured materials for applications in nano-medicine, cultural heritage, and electrochemical biosensors. Anal Bioanal Chem 405: 451-465, 2013.

8. Alexis F, Rhee JW, Richie JP, Radovic-Moreno AF, Langer R and Farokhzad OC: New frontiers in nanotechnology for cancer treatment. Urol Oncol 26: 74-85, 2008.

9. Chen SL, Fang WW, Ye F, Liu YH, Qian J, Shan SJ, Zhang JJ, Chunhua RZ, Liao LM, Lin S, et al: Effect on left ventricular function of intracoronary transplantation of autologous bone marrow mesenchymal stem cell in patients with acute myocardial infarction. Am J Cardiol 94: 92-95, 2004.

10. Bang OY, Lee JS, Lee PH and Lee G: Autologous mesenchymal stem cell transplantation in stroke patients. Ann Neurol 57: 874-882, 2005. 
11. Kuo TK, Hung SP, Chuang CH, Chen CT, Shih YR, Fang SC, Yang VW and Lee OK: Stem cell therapy for liver disease: Parameters governing the success of using bone marrow mesenchymal stem cells. Gastroenterology 134: 2111-2121, 2121.e1-2121.e3, 2008.

12. Shi Y, Moon M, Dawood S, McManus B and Liu PP: Mechanisms and management of doxorubicin cardiotoxicity. Herz 36: 296-305, 2011.

13. Poletti V, Casoni GL, Cancellieri A, Piciucchi S, Dubini A and Zompatori M: Diffuse alveolar damage. Pathologica 102: 453-463, 2010.

14. Naranjo TW, Lopera DE, Diaz-Granados LR, Duque JJ, Restrepo AM and Cano LE: Combined itraconazole-pentoxifylline treatment promptly reduces lung fibrosis induced by chronic pulmonary paracoccidioidomycosis in mice. Pulm Pharmacol Ther 24: 81-91, 2011.

15. de Pascual-Teresa S, Santos-Buelga $\mathrm{C}$ and Rivas-Gonzalo JC: Quantitative analysis of flavan-3-ols in Spanish foodstuffs and beverages. J Agric Food Chem 48: 5331-5337, 2000.

16. Li X, Chen D, Wang G and Lu Y: Study of interaction between human serum albumin and three antioxidants: Ascorbic acid, $\alpha$-tocopherol, and proanthocyanidins. Eur J Med Chem 70 22-36, 2013.

17. Wiesneth S, Petereit F and Jürgenliemk G: Salix daphnoides: A Screening for Oligomeric and Polymeric Proanthocyanidins. Molecules 20: 13764-13779, 2015.

18. Fernández K, Vega M and Aspé E: An enzymatic extraction of proanthocyanidins from País grape seeds and skins. Food Chem 168: 7-13, 2015.

19. Roopchand DE, Krueger CG, Moskal K, Fridlender B, Lila MA and Raskin I: Food-compatible method for the efficient extraction and stabilization of cranberry pomace polyphenols. Food Chem 141: 3664-3669, 2013.

20. Lombardo Bedran TB, Palomari Spolidorio D and Grenier D: Green tea polyphenol epigallocatechin-3-gallate and cranberry proanthocyanidins act in synergy with cathelicidin (LL-37) to reduce the LPS-induced inflammatory response in a three-dimensional co-culture model of gingival epithelial cells and fibroblasts. Arch Oral Biol 60: 845-853, 2015.

21. Pons Z, Guerrero L, Margalef M, Arola L, Arola-Arnal A and Muguerza B: Effect of low molecular grape seed proanthocyanidins on blood pressure and lipid homeostasis in cafeteria diet-fed rats. J Physiol Biochem 70: 629-637, 2014.

22. Zhang XY, Li WG, Zheng TZ and Li W: Effects of proanthocyanidins on contractile activity of aortic smooth muscle and platelet aggregation in experimental animals. Zhongguo Ying Yong Sheng Li Xue Za Zhi 21: 383-386, 2005 (In Chinese).

23. Mohana T, Navin AV, Jamuna S, Sakeena Sadullah MS and Niranjali Devaraj S: Inhibition of differentiation of monocyte to macrophages in atherosclerosis by oligomeric proanthocyanidins -In-vivo and in-vitro study. Food Chem Toxicol 82: 96-105, 2015.

24. de Sá M, Justino V, Spranger MI, Zhao YQ, Han L and Sun BS: Extraction yields and anti-oxidant activity of proanthocyanidins from different parts of grape pomace: Effect of mechanical treatments. Phytochem Anal 25: 134-140, 2014

25. Luan YY, Liu ZM, Zhong JY, Yao RY and Yu HS: Effect of grape seed proanthocyanidins on tumor vasculogenic mimicry in human triple-negative breast cancer cells. Asian Pac J Cancer Prev 16: 531-535, 2015.

26. D’Angelo L, Piazzi G, Pacilli A, Prossomariti A, Fazio C, Montanaro L, Graziani G, Fogliano V, Munarini A, Bianchi F et al: A combination of eicosapentaenoic acid-free fatty acid, epigallocatechin-3-gallate and proanthocyanidins has a strong effect on mTOR signaling in colorectal cancer cells. Carcinogenesis 35: 2314-2320, 2014.

27. Huang S, Yang N, Liu Y, Gao J, Huang T, Hu L, Zhao J, Li Y, Li C and Zhang X: Grape seed proanthocyanidins inhibit colon cancer-induced angiogenesis through suppressing the expression of VEGF and Ang1. Int J Mol Med 30: 1410-1416, 2012.

28. Sharma SD and Katiyar SK: Dietary grape seed proanthocyanidins inhibit UVB-induced cyclooxygenase-2 expression and other inflammatory mediators in UVB-exposed skin and skin tumors of SKH-1 hairless mice. Pharm Res 27: 1092-1102, 2010.

29. Zhang XY, Li WG, Wu YJ and Gao MT: Amelioration of doxorubicin-induced myocardial oxidative stress and immunosuppression by grape seed proanthocyanidins in tumour-bearing mice. J Pharm Pharmacol 57: 1043-1052, 2005.

30. Shen S, Kepp O, Michaud M, Martins I, Minoux H, Métivier D, Maiuri MC, Kroemer RT and Kroemer G: Association and dissociation of autophagy, apoptosis and necrosis by systematic chemical study. Oncogene 30: 4544-4556, 2011.
31. Chiantore MV, Vannucchi S, Mangino G, Percario ZA, Affabris E, Fiorucci G and Romeo G: Senescence and cell death pathways and their role in cancer therapeutic outcome. Curr Med Chem 16: 287-300, 2009.

32. Klionsky DJ: Autophagy: From phenomenology to molecular understanding in less than a decade. Nat Rev Mol Cell Biol 8: 931-937, 2007.

33. Levine B and Kroemer G: Autophagy in the pathogenesis of disease. Cell 132: 27-42, 2008

34. Wang MC, Wu AG, Huang YZ, Shao GL, Ji SF, Wang RW, Yuan HJ, Fan XL, Zheng LH and Jiao QL: Autophagic regulation of cell growth by altered expression of Beclin 1 in triple-negative breast cancer. Int J Clin Exp Med 8: 7049-7058, 2015.

35. Sui H, Shi C, Yan Z and Li H: Combination of erlotinib and a PARP inhibitor inhibits growth of A2780 tumor xenografts due to increased autophagy. Drug Des Devel Ther 9: 3183-3190, 2015

36. Zheng JF, Li LL, Lu J, Yan K, Guo WH and Zhang JX: XPD functions as a tumor suppressor and dysregulates autophagy in cultured HepG2 cells. Med Sci Monit 21: 1562-1568, 2015.

37. Rosenfeldt MT and Ryan KM: The multiple roles of autophagy in cancer. Carcinogenesis 32: 955-963, 2011.

38. Duan Y, Ke J, Zhang H, He Y, Sun G and Sun X: Autophagic cell death of human hepatoma $\mathrm{G} 2$ cells mediated by procyanidins from Castanea mollissima Bl. Shell-induced reactive oxygen species generation. Chem Biol Interact 224: 13-23, 2014

39. Mizushima N: Methods for monitoring autophagy. Int J Biochem Cell Biol 36: 2491-2502, 2004.

40. Eskelinen EL and Saftig P: Autophagy: A lysosomal degradation pathway with a central role in health and disease. Biochim Biophys Acta 1793: 664-673, 2009.

41. He H, Dang Y, Dai F, Guo Z, Wu J, She X, Pei Y, Chen Y, Ling W, Wu C, et al: Post-translational modifications of three members of the human MAP1LC3 family and detection of a novel type of modification for MAP1LC3B. J Biol Chem 278: 29278-29287, 2003.

42. Zheng HY, Zhang XY, Wang XF and Sun BC: Autophagy enhances the aggressiveness of human colorectal cancer cells and their ability to adapt to apoptotic stimulus. Cancer Biol Med 9: 105-110, 2012

43. Gozuacik D and Kimchi A: Autophagy as a cell death and tumor suppressor mechanism. Oncogene 23: 2891-2906, 2004.

44. Zhang Z, Zheng L, Zhao Z, Shi J, Wang X and Huang J: Grape seed proanthocyanidins inhibit $\mathrm{H}_{2} \mathrm{O}_{2}$-induced osteoblastic MC3T3-E1 cell apoptosis via ameliorating $\mathrm{H}_{2} \mathrm{O}_{2}$-induced mitochondrial dysfunction. J Toxicol Sci 39: 803-813, 2014.

45. Chen Q, Liu XF and Zheng PS: Grape seed proanthocyanidins (GSPs) inhibit the growth of cervical cancer by inducing apoptosis mediated by the mitochondrial pathway. PLoS One 9: e107045, 2014

46. Prasad R, Vaid M and Katiyar SK: Grape proanthocyanidin inhibit pancreatic cancer cell growth in vitro and in vivo through induction of apoptosis and by targeting the PI3K/Akt pathway. PLoS One 7: e43064, 2012.

47. Kim ME, Ha TK, Yoon JH and Lee JS: Myricetin induces cell death of human colon cancer cells via BAX/BCL2-dependent pathway. Anticancer Res 34: 701-706, 2014.

48. Choi YJ, Saez B, Anders L, Hydbring P, Stefano J, Bacon NA, Cook C, Kalaszczynska I, Signoretti S, Young RA, et al: D-cyclins repress apoptosis in hematopoietic cells by controlling death receptor Fas and its ligand FasL. Dev Cell 30: 255-267, 2014.

49. Yao W, Yue P, Zhang G, Owonikoko TK, Khuri FR and Sun SY: Enhancing therapeutic efficacy of the MEK inhibitor, MEK162, by blocking autophagy or inhibiting PI3K/Akt signaling in human lung cancer cells. Cancer Lett 364: 70-78, 2015.

50. Xin $M$ and Deng X: Nicotine inactivation of the proapoptotic function of Bax through phosphorylation. J Biol Chem 280: 10781-10789, 2005

51. Song G, Ouyang G and Bao S: The activation of Akt/PKB signaling pathway and cell survival. J Cell Mol Med 9: 59-71, 2005.

52. Pan ST, Qin Y, Zhou ZW, He ZX, Zhang X, Yang T, Yang YX, Wang D, Qiu JX and Zhou SF: Plumbagin induces $\mathrm{G}_{2} / \mathrm{M}$ arrest, apoptosis, and autophagy via p38 MAPK- and PI3K/Akt/mTORmediated pathways in human tongue squamous cell carcinoma cells. Drug Des Devel Ther 9: 1601-1626, 2015

53. Zhou ZW, Li XX, He ZX, Pan ST, Yang Y, Zhang X, Chow K, Yang T, Qiu JX, Zhou Q, et al: Induction of apoptosis and autophagy via sirtuin1- and PI3K/Akt/mTOR-mediated pathways by plumbagin in human prostate cancer cells. Drug Des Devel Ther 9: 1511-1554, 2015. 
54. Yuan L, Wei S, Wang J and Liu X: Isoorientin induces apoptosis and autophagy simultaneously by reactive oxygen species (ROS)-related p53, PI3K/Akt, JNK, and p38 signaling pathways in HepG2 cancer cells. J Agric Food Chem 62: 5390-5400, 2014.

55. Zhang H, Guo M, Chen JH, Wang Z, Du XF, Liu PX and Li WH: Osteopontin knockdown inhibits $\alpha \mathrm{v}, \beta 3$ integrin-induced cell migration and invasion and promotes apoptosis of breast cancer cells by inducing autophagy and inactivating the PI3K/Akt/ mTOR pathway. Cell Physiol Biochem 33: 991-1002, 2014.

56. Hu Y, Li L, Yin W, Shen L, You B and Gao H: Protective effect of proanthocyanidins on anoxia-reoxygenation injury of myocardial cells mediated by the PI3K/Akt/GSK-3 $\beta$ pathway and mitochondrial ATP-sensitive potassium channel. Mol Med Rep 10: 2051-2058, 2014.

57. Zhong Y, Wang QJ and Yue Z: Atg14L and Rubicon: Yin and yang of Beclin 1-mediated autophagy control. Autophagy 5: 890-891, 2009.

58. Liang XH, Jackson S, Seaman M, Brown K, Kempkes B, Hibshoosh $\mathrm{H}$ and Levine B: Induction of autophagy and inhibition of tumorigenesis by beclin 1. Nature 402: 672-676, 1999.

59. Baspinar S, Bircan S, Orhan H, Kapucuoglu N and Bozkurt KK: The relation of beclin 1 and bcl-2 expressions in high grade prostatic intraepithelial neoplasia and prostate adenocarcinoma: A tissue microarray study. Pathol Res Pract 210: 412-418, 2014.

60. Fukui M, Yamabe N, Choi HJ, Polireddy K, Chen Q and Zhu BT: Mechanism of ascorbate-induced cell death in human pancreatic cancer cells: Role of Bcl-2, Beclin 1 and autophagy. Planta Med 81: 838-846, 2015.
61. Tian PG, Jiang ZX, Li JH, Zhou Z and Zhang QH: Spliced XBP1 promotes macrophage survival and autophagy by interacting with Beclin-1. Biochem Biophys Res Commun 463: 518-523, 2015.

62. Patro SC, Pal S, Bi Y, Lynn K, Mounzer KC, Kostman JR, Davuluri RV and Montaner LJ: Shift in monocyte apoptosis with increasing viral load and change in apoptosis-related ISG/Bcl2 family gene expression in chronically HIV-1-infected subjects. J Virol 89: 799-810, 2015.

63. Chaudhary P and Vishwanatha JK: c-Jun NH2-terminal kinase-induced proteasomal degradation of c-FLIPL/S and Bcl2 sensitize prostate cancer cells to Fas- and mitochondriamediated apoptosis by tetrandrine. Biochem Pharmacol 91: 457-473, 2014.

64. Lin W and Tongyi S: Role of Bax/Bcl-2 family members in green tea polyphenol induced necroptosis of p53-deficient Hep3B cells. Tumour Biol 35: 8065-8075, 2014.

65. Hua F, Cornejo MG, Cardone MH, Stokes CL and Lauffenburger DA: Effects of Bcl-2 levels on Fas signalinginduced caspase-3 activation: Molecular genetic tests of computational model predictions. J Immunol 175: 985-995, 2005.

66. Park SE, Shin WT, Park C, Hong SH, Kim GY, Kim SO, Ryu CH, Hong SH and Choi YH: Induction of apoptosis in MDA-MB-231 human breast carcinoma cells with an ethanol extract of Cyperus rotundus L. by activating caspases. Oncol Rep 32: 2461-2470, 2014. 